\title{
Use of electronic medical records to describe general practitioner antibiotic prescribing patterns
}

Lesley Hawes, Lyle Turner, Kirsty Buising, Danielle Mazza

\section{Background and objectives}

The computerised medical records of general practice patients can inform our understanding of antibiotic prescribing and assist in antimicrobial stewardship (AMS). The aim of this study was to describe Australian general practitioner (GP) antibiotic prescribing patterns using data extracted from electronic medical records (EMR).

\section{Method}

A descriptive analysis of patient records from 44 general practices, between 2010 and 2014, in the eastern region of metropolitan Melbourne was undertaken.

\section{Results}

Of the 615,362 antibiotic prescriptions, cefalexin, amoxicillin-clavulanic acid, roxithromycin, doxycycline and clarithromycin were the most frequently prescribed antibiotics. Except for cefalexin, prescribing rates of the antibiotics increased in winter. Of 472,197 patients consulting a GP in one of these practices, $34.8 \%$ received an antibiotic at some point over the five years. There was a higher rate of prescribing per consultation in patients aged $<20$ years.

\section{Discussion}

This study shows that it is possible to examine EMR for antibiotic prescriptions, and that a descriptive analysis can identify AMS targets.
IN 2014, $46 \%$ of Australians received at least one antibiotic prescription. ${ }^{1}$ This prescribing rate is higher per capita than in many comparable countries, including England, Canada, Sweden and the Netherlands. ${ }^{1}$ The volume prescribed is higher than the Organisation for Economic Co-operation and Development average. ${ }^{2}$ The difference is considerable, with consumption in Australia apparently twice that of Canada and three times that of Sweden. ${ }^{1}$ There are no obvious reasons why antibiotic use should be higher in the Australian community setting; therefore, it is likely that some antibiotic use may be unnecessary.

Antibiotic use exposes patients to the risk of adverse effects and the development of resistance. This may include drug side effects and the development of antibiotic resistance among bacteria in that individual and in the population more broadly. ${ }^{3}$ Antimicrobial stewardship (AMS) aims to guide the use of antimicrobial drugs to optimise patient outcomes while minimising any adverse effects. Most human antimicrobial use is from antibiotics prescribed in primary care; therefore, general practitioners (GPs) have a critical role in AMS. ${ }^{4}$

To address AMS, we need accurate data on antibiotic prescribing by Australian GPs, but this is not available from current data sources. Three main sources of data are the Pharmaceutical Benefits Scheme (PBS)/Repatriation Pharmaceutical Benefits Scheme, the MedicineInsight program and the Bettering the Evaluation and Care of Health (BEACH) survey, but each has significant limitations. PBS data are administrative data with no clinical information. Up to $25 \%$ of PBS prescriptions are from non-GP providers, and private prescriptions (outside the PBS restrictions) are not included. ${ }^{5}$ The MedicineInsight program, ${ }^{6}$ managed by NPS MedicineWise, aggregates data from GP electronic medical records (EMR) for reports as required. ${ }^{1}$ While Australia-wide, it is a voluntary program with antibiotic prescriptions only one part of its remit. BEACH was a paper-based survey of 100 consecutive patient presentations from a representative sample of 1000 GPs per year. It collected data including problems managed and medications prescribed, but was discontinued in 2016. While the extensive data are still available, the survey cannot be used for ongoing monitoring. ${ }^{7}$

A range of clinical software is used in Australian general practices. As a result of limited interoperability, this has limited the secondary analysis of EMR. ${ }^{8}$ There is a need to develop a sustainable, ongoing way to accurately monitor GP prescribing and to meaningfully interpret the data available.

The aim of this research was to extract routinely collected data from general practice EMR and to use this to describe antibiotic prescribing patterns.

\section{Method}

We retrospectively analysed routinely collected GP data from the EMR of patients from 50 general practices across Melbourne's inner eastern suburbs using POLAR (Population Level Analysis and Reporting for general practice formerly known as MAGNET). ${ }^{9}$

Patient-level data were extracted for consultations and antibiotic prescriptions between 1 January 2010 and 31 December 2014. Unique linkage 
keys linked consultation data with antibiotic prescriptions and could track patients across practices. Consultation data included dates of consultations and the age of the patient. Antibiotics were identified and coded according to the Anatomical Therapeutic Chemical Classification. ${ }^{10}$ Only the original prescription was included; repeats on the same prescription were not examined as the majority of repeat antibiotic prescriptions were not dispensed. ${ }^{5}$ Systemic and topical antibiotic prescriptions were included in the analysis, except for topical chloramphenicol, which is available without prescription in Australia. During this pilot research, some links between the consultation data and the antibiotic data were inadvertently broken. Where the antibiotic prescription did not link directly to a consultation, the previous or subsequent consultation in that year was used to determine the patient's age at time of consultation. If there was no other consultation in that year by the patient, or no age was available, the age was recorded as missing. Ages were examined in four broad ranges: under 1, 1-19, 20-49 and $\geq 50$.

Descriptive analysis of the data was undertaken using Stata 13.1 (StataCorp). We examined the numbers of each antibiotic prescribed and the number of consultations. Antibiotic prescribing was assessed across the days of the week, months and years and by the age of the patient.

The Monash University Human Research Ethics Committee granted ethics approval for this research(number CF12/1057-2012000504).

\section{Results}

Antibiotic prescribing data were complete for 44 of the 50 practices, with 615,362 antibiotic prescriptions provided to 166,772 patients over the five years. Ten antibiotics accounted for 518,016 (84.2\%) of the antibiotic prescriptions (Table 1). Cefalexin $(146,155,23.8 \%)$, was the most frequently prescribed antibiotic. The number of cefalexin prescriptions remained constant over the five-year period (Figure 1). Trimethoprim and metronidazole, the sixth and tenth most commonly prescribed antibiotics respectively (Table 1), also remained constant over time (Figure 2). By contrast, the remaining seven most commonly prescribed antibiotics - amoxicillin-clavulanic acid, roxithromycin, doxycycline, clarithromycin (Figure 1), cefaclor, erythromycin, and phenoxymethylpenicillin (not shown) - had prescribing peaks in winter. Amoxicillin-clavulanic acid $(93,380$, $15.2 \%)$ was more commonly prescribed than amoxicillin $(7390,1.2 \%)$ and other forms of penicillin (Table 1). Phenoxymethylpenicillin (22,090, 3.6\%) was the only narrow-spectrum beta-lactam antibiotic in the ten most frequently prescribed antibiotics (Table 1). The number of prescriptions and winter peaks for roxithromycin (Figure 1), cefaclor and erythromycin declined over time, but they were still being prescribed in large numbers in 2014. The most commonly prescribed quinolone was norfloxacin, with 9843 prescriptions over five years (1.6\% of total; Table 1$)$. Macrolides (roxithromycin, clarithromycin and erythromycin) comprised 129,132 (21\%) prescriptions (Table 1).

Consultation data were complete for 39 of the above 44 practices, with 6,227,104 recorded consultations in the five-year period involving 472,197 patients. A total of $164,522(34.8 \%)$ of the patients received 597,302 antibiotic prescriptions.

The antibiotic prescribing rate per consultation varied with age, which was available for 590,105 (98.8\%) of the prescriptions. Patients aged 1-19 years had fewer consultations but received antibiotics at a higher rate per consultation than infants aged $<1$ and adults aged $>19$ years. The rate of antibiotic prescribing per consultation per year fell between 2010 and 2014 in age groups $\leq 49$ years, with the largest declines occurring in age group $1-19$ years. Age group $\geq 50$ years showed little variation in the antibiotic prescribing rate per consultation over time. Cefalexin was the most prescribed antibiotic across all age groups.

Examination of the reason-forprescription field (which did not include the progress notes) revealed that for

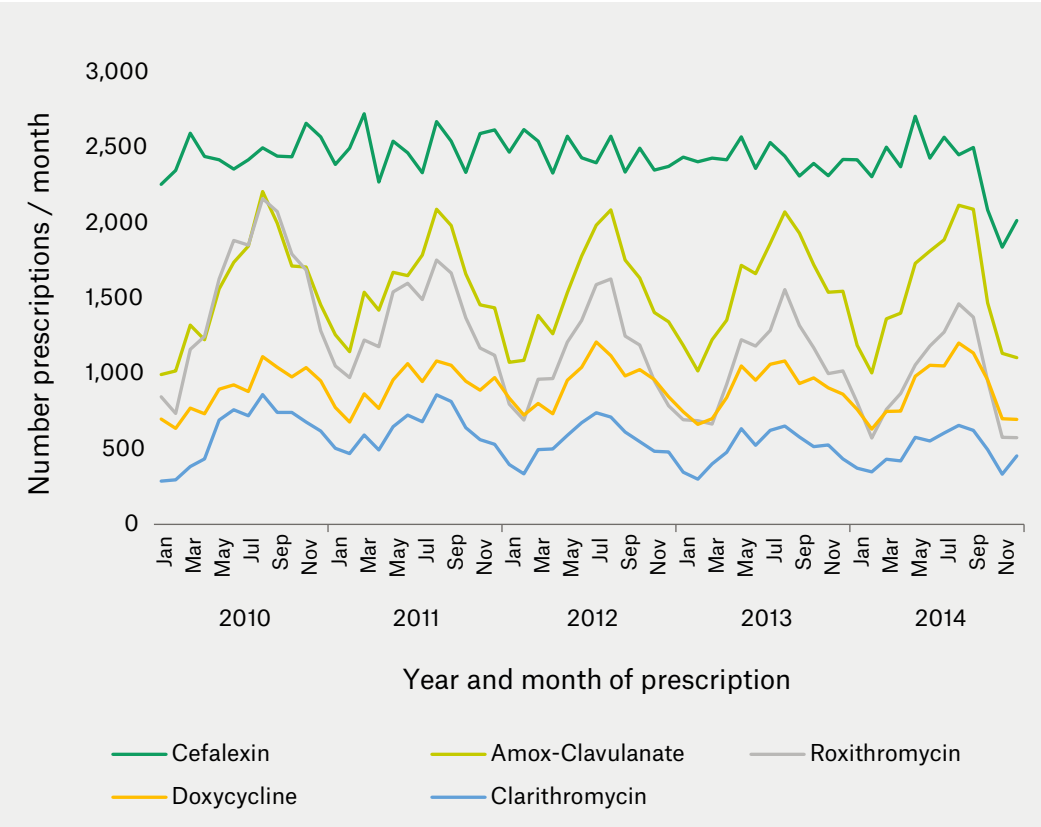

Figure 1. The five most frequently prescribed antibiotics by year and month of prescription (44 practices)

Note: Summer is December to February and winter is June to August. 
494,085 (82.7\%) of the prescriptions, no reason was available.

\section{Discussion}

This research shows the utility of data extracted from general practice EMRs and that a descriptive analysis, using this cohort as the example, can provide information to direct AMS activities.

Not only is the Australian prescribing rate high in comparison to European countries, ${ }^{1,2}$ with most antibiotics prescribed by GPs, ${ }^{11}$ but there is a preference for broad-spectrum antibiotic agents in this cohort. In 2013, on the basis of PBS data, amoxicillin, cefalexin and amoxicillin-clavulanic acid were the most dispensed antibiotics Australia-wide. ${ }^{5}$ However, amoxicillin accounted for only
$1.2 \%$ of the total antibiotic use in this cohort. Explanations for this cannot be obtained from the available data. Why this cohort of practices showed such low rates of penicillin, amoxicillin and flucloxacillin prescribing and high rates of cefalexin and amoxicillin-clavulanic acid prescribing needs further investigation to understand the clinical and non-clinical drivers. Macrolides were commonly prescribed. This local preference for broad-spectrum antibiotics contrasts with self-reported intentions of Australian GPs, with $70 \%$ reporting in 2013 that they would always/often prescribe narrowspectrum antibiotics. ${ }^{12}$ It is possible that GPs may not perceive cephalosporins, amoxicillin-clavulanic acid and macrolides to be broad-spectrum agents. In 2012 in Europe, narrow-spectrum penicillins

Table 1. Antibiotics by frequency of prescription 2010-14 (44 practices, $\mathrm{n}=615,362$ )

\begin{tabular}{lcc}
\hline Antibiotic & Frequency & Percentage \\
\hline Cefalexin & 146,155 & 23.8 \\
\hline Amoxicillin-Clavulanic acid & 93,380 & 15.2 \\
\hline Roxithromycin & 72,089 & 11.7 \\
\hline Doxycycline & 54,389 & 8.8 \\
\hline Clarithromycin & 33,215 & 5.4 \\
\hline Trimethoprim & 27,679 & 4.5 \\
\hline Cefaclor & 24,354 & 4.0 \\
\hline Erythromycin & 23,828 & 3.9 \\
\hline Phenoxymethylpenicillin & 22,090 & 3.6 \\
\hline Metronidazole & 20,837 & 3.4 \\
\hline Mupirocin & 18,899 & 3.0 \\
\hline Framycetin & 14,062 & 2.3 \\
\hline Flucloxacillin & 12,719 & 2.1 \\
\hline Norfloxacin & 9,843 & 1.6 \\
\hline Amoxicillin & 7,390 & 1.2 \\
\hline Minidazole & 6,691 & 2.6 \\
\hline Orimocycline & 6,163 & 15,990 \\
\hline
\end{tabular}

were the most frequently used group of antibiotics in the community. ${ }^{13,14}$ Infections encountered in community practice in Europe would be expected to be similar to those in Australia, and therefore it would be expected to be safe to use these for many common conditions (informed by any local differences in pathogen resistance patterns). Promotion of narrowspectrum penicillins could be a local AMS target. The prescription of quinolones is restricted in Australia, and no quinolone was represented in the ten most frequently prescribed antibiotics.

The lack of seasonality in prescribing of cefalexin, trimethoprim and metronidazole probably reflects use in year-round infections such as skin, urinary tract, genital and intestinal infections. Of note, the Therapeutic Guidelines recommended flucloxacillin as the firstline antibiotic for skin and soft tissue infection; ${ }^{15}$ however, it was not among the most commonly prescribed antibiotics in these practices.

The winter prescribing peaks suggest prescribing for respiratory tract infections, which has been described in Australian ${ }^{16}$ and international studies. ${ }^{17,18}$ During 2010, Therapeutic Guidelines ceased recommending the use of cefaclor and roxithromycin for pneumonia, but in 2014 they were still being prescribed with a winter peak frequency. It suggests that this cohort of prescribers had either incomplete awareness of the changed guideline or used different guidelines. Therapeutic Guidelines did not recommend amoxicillin-clavulanic acid for community respiratory tract infections, ${ }^{15}$ but the winter peaks in prescribing suggest that it was being prescribed for this reason. A study of which guidelines these GPs use and how guideline changes are notified to them seems indicated.

The 2014 PBS figure of $46 \%$ of the Australian population being dispensed at least one antimicrobial agent per year ${ }^{1}$ is higher than the $34.8 \%$ of patients in this cohort. ${ }^{19}$ This may be due to PBS including dispensing data from other providers (such as community-based specialists, emergency department and private hospital inpatients). ${ }^{5}$ MedicineInsight data (a larger Australia-wide general practice 
electronic medical record extraction program) showed that $30 \%$ of patients were prescribed systematic antimicrobials in 2014. ${ }^{1}$ This is comparable to our data, which included topical antibiotics.

The reason for the decline in prescribing rate between 2010 and 2014 in age groups $<20$ years is unknown. It may relate to a decline in general practice presentations for upper respiratory tract infections, throat complaints and ear pain/earache between 2006-07 and $2015-16^{7}$ or the fall in cefaclor and roxithromycin prescriptions seen in this study. Community campaigns during this period were discouraging antibiotics for common colds, which may have influenced behaviour. This would require a more detailed investigation.

This research examined routinely collected data from the EMR of general practice patients. In Australia, patients may attend any general practice; an advantage of POLAR is that it could track patients across practices within the POLAR catchment. A major limitation is that the data extracted are entirely dependent on the clinical software package and what GPs chose to document and where. ${ }^{20}$ The reason-for-prescription field in some software packages was free text and in all packages was optional, resulting in a low completion rate. We were unable to determine the reasons for the antibiotic prescriptions and note that antibiotics may be prescribed for common conditions such as acne rosacea or acne vulgaris and for prophylaxis (eg malaria). This limitation requires information technology and standardisation solutions beyond the scope of this research.

However, despite this serious limitation, we have shown how data available in general practice EMR might be used for AMS. Of the 50 practices in the dataset, six did not have a complete medication dataset, and a further five practices had incomplete consultation data. This may be due to software changes/updates between entry and extraction, ${ }^{8,21}$ and requires further investigation. This dataset represents the doctors and patients in a defined urban area, so may not be typical of other regions. However, this may facilitate targeting of AMS initiatives to specific practices or defined localities.
Comorbidities were not examined in this study but would be a valuable addition to future studies. The data presented here are antibiotic prescriptions written by GPs. There is no linkage with dispensed data, so it is not known how many of these prescriptions may have been delayed prescriptions provided to a patient with instructions of only filling the prescription if the patient's condition deteriorated, or if a patient chose not to fill the prescription.

\section{Implications for general practice}

This study shows that Australian general practice EMR data can be extracted, and that a descriptive analysis of antibiotic prescriptions can identify targets for intervention in AMS programs and monitor change over time. For example, in this cohort, we revealed high use of broad-spectrum antibiotics and winter prescribing peaks. The continued prescribing of cefaclor and roxithromycin in winter peaks suggests incomplete awareness of changes in Therapeutic Guidelines for treatment of respiratory infections, or the use of alternative guidelines. Information for AMS would be significantly enhanced if reasons for prescription were documented in the EMR in a standardised field. GPs should be encouraged to complete the reason-forprescription field. Software changes are required to improve data capture at the GP-software interface. GPs and Primary Health Networks should be encouraged to conduct AMS audits of antibiotic prescriptions from EMR.

\section{Authors \\ Lesley Hawes BSc (Hons), GCUT, MPH, MASM, PhD Fellow, Department of General Practice, Monash University; and National Centre for Antimicrobial Stewardship, Melbourne, Vic. Lesley.Hawes@monash.edu \\ Lyle Turner BSc (Hons), PhD, Manager, Data and Research Unit, Institute for Urban Indigenous Health, Qld; Department of General Practice, School of Primary and Allied Health Care, Monash University, Clayton, Vic Kirsty Buising MBBS, MD, MPH, FRACP, Deputy Director, National Centre for Antimicrobial Stewardship; Infectious diseases physician, Victorian Infectious Diseases Service, Royal Melbourne Hospital, Vic \\ Danielle Mazza MD, MBBS, FRACGP, DRANZCOG, Grad Dip Women's Health, GAICD, Head, Department of General Practice, School of Primary and Allied Health Care, Faculty of Medicine Nursing and Health Sciences, Monash University; Conjoint Professor, School of Medicine and Public Health, University of Newcastle; Fellow, Society of Family Planning, USA; National Centre for Antimicrobial Stewardship, The Peter Doherty Institute for Infection and Immunity, Melbourne, Vic}

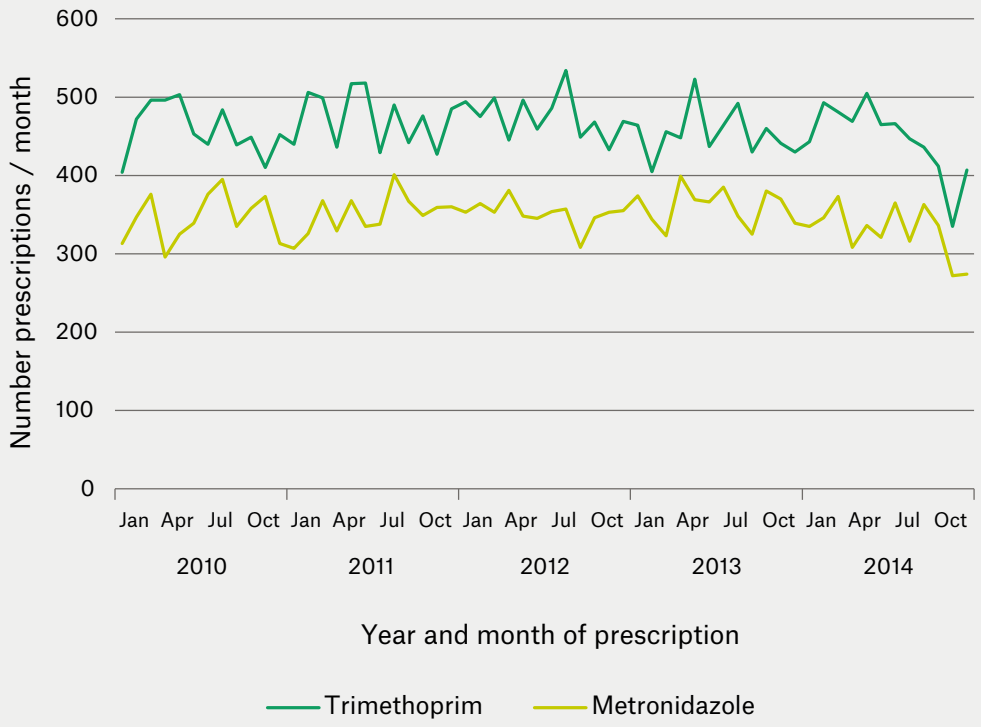

Figure 2. Trimethoprim and metronidazole prescriptions by year and month of prescription (6th and 10th most frequently prescribed) 
Competing interests: None.

Funding: This work was funded by the National Health and Medical Research Council (National Centre for Antimicrobial Stewardship - Grant No. 1079625) LH received an RTP stipend.

Provenance and peer review: Not commissioned, externally peer reviewed.

\section{Acknowledgements}

Outcome Health for access to the MAGNET/POLAR data and the GPs and practices who participated in the MAGNET/POLAR research project.

\section{References}

1. Australian Commission on Safety and Quality in Health Care. AURA 2016: First Australian report on antimicrobial use and resistance in human health. Sydney: ACSQHC, 2016.

2. OECD. Health at a Glance 2015: OECD indicators. Paris: OECD Publishing, 2015.

3. Costelloe C, Metcalfe C, Lovering A, Mant D, Hay AD. Effect of antibiotic prescribing in primary care on antimicrobial resistance in individual patients: Systematic review and meta-analysis. BMJ 2010;340:c2096. doi: 10.1136/bmj.c2096.

4. World Health Organization. WHO global strategy for containment of antimicrobial resistance. Geneva: WHO, 2001.

5. Pharmaceutical Benefits Advisory Committee, Drug utilisation sub-committee. Antibiotics: PBS/RPBS utilisation, Oct 2014 and Feb 2015. Canberra: Department of Health, 2015.

6. NPS MedicineWise. Medicinelnsight: Improving clinical practice and health outcomes for Australians. Available at www.nps.org.au/ medicine-insight [Accessed 1 April 2018].

7. Britt H, Miller GC, Bayram C, et al. A decade of Australian general practice activity 2006-07 to 2015-16. General practice series no 41. Sydney: Sydney University Press, 2016

8. Liaw ST, Taggart J, Dennis S, Yeo A. Data quality and fitness for purpose of routinely collected data - A general practice case study from an electronic practice-based research network (ePBRN). AMIA Annu Symp Proc 2011;2011:785-94.

9. Mazza D, Pearce C, Turner LR, et al. The Melbourne East Monash General Practice Database (MAGNET): Using data from computerised medical records to create a platform for primary care and health services research. J Innov Health Inform 2016;23(2):181. doi: 10.14236/ jhi.v23i2.181.

10. WHO Collaborating Centre for Drug Statistics Methodology. ATC/DDD index 2018. Available at www.whocc.no/atc_ddd_index/[Accessed 5 October 2018].

11. Australian Government. Antimicrobial resistance. Canberra: Commonwealth of Australia, 2017. Available at www.amr.gov.au [Accessed 20 August 2018].

12. Hardy-Holbrook R, Aristidi S, Chandnani $V$ DeWindt D, Dinh K. Antibiotic resistance and prescribing in Australia: Current attitudes and practice of GPs. Healthcare infection 2013;18(4):147-51. doi: https://doi.org/10.1071/ HI13019.

13. Danish Integrated Antimicrobial Resistance Monitoring and Research Programme. DANMAP 2012: Use of antimicrobial agents and occurrence of antimicrobial resistance in bacteria from food animals, food and humans in Denmark. Copenhagen: DANMAP, 2013.
14. Public Health Agency of Sweden; National Veterinary Institute. Use of antimicrobials and occurrence of antimicrobial resistance in Sweden. Solna/Uppsala, Sweden: SWEDRESSVARM, 2013.

15. Expert Groups for Antibiotic. Antibiotics: Skin and soft tissue infections: Bacterial. In: eTG complete [intenet]. Melbourne: Therapeutic Guidelines Ltd, 2018.

16. McCullough AR, Pollack AJ, Plejdrup Hansen M, et al. Antibiotics for acute respiratory infections in general practice: Comparison of prescribing rates with guideline recommendations. Med J Aust 2017;207(2):65-69.

17. Goossens $H$, Ferech $M$, Vander Stichele RV, Elseviers M, ESAC Project Grp. Outpatient antibiotic use in Europe and association with resistance: A cross-national database study. Lancet 2005;365(9459):579-87. doi: 10.1016/ S0140-6736(05)17907-0.

18. Sun L, Klein EY, Laxminarayan R. Seasonality and temporal correlation between community antibiotic use and resistance in the United States. Clin Infect Dis 2012;55(5):687-94. doi: 10.1093/ cid/cis509.

19. Australian Commission on Safety and Quality in Health Care, National Health Performance Authority. Australian atlas of healthcare variation. Sydney: ACSQHC, 2015.

20. Liaw ST, Powell-Davies G, Pearce C, Britt H, McGlynn L, Harris MF. Optimising the use of observational electronic health record data: Current issues, evolving opportunities, strategies and scope for collaboration. Aust Fam Physician 2016;45(3):153-56.

21. Gordon J, Miller G, Britt H. Reality check - Reliable national data from general practice electronic health records. Canberra: Deeble Institute, 2016. 